\title{
Une approche ethnolinguistique sur le lexique de l'élevage chez les Jbala et les Ghomara (nord-ouest du Maroc)
}

An Ethnolinguistic Approach about the Lexicon of Livestock in Jbala and Ghomara (Northwestern Morocco)

\section{Amina Naciri-Azzouz et Ángeles Vicente}

\section{OpenEdition \\ Journals}

Édition électronique

URL : https://journals.openedition.org/ethnoecologie/3146

DOI : 10.4000/ethnoecologie.3146

ISSN : 2267-2419

Éditeur

Laboratoire Éco-anthropologie

\section{Référence électronique}

Amina Naciri-Azzouz et Ángeles Vicente, « Une approche ethnolinguistique sur le lexique de l'élevage chez les Jbala et les Ghomara (nord-ouest du Maroc) », Revue d'ethnoécologie [En ligne], Supplément 1 | 2017, mis en ligne le 17 octobre 2017, consulté le 19 novembre 2021. URL : http://

journals.openedition.org/ethnoecologie/3146; DOI : https://doi.org/10.4000/ethnoecologie.3146

Ce document a été généré automatiquement le 19 novembre 2021.

\section{cc) (†)}

Revue d'ethnoécologie est mis à disposition selon les termes de la licence Creative Commons Attribution - Pas d'Utilisation Commerciale - Pas de Modification 4.0 International. 


\title{
Une approche ethnolinguistique sur le lexique de l'élevage chez les Jbala et les Ghomara (nord-ouest du
} Maroc)

\author{
An Ethnolinguistic Approach about the Lexicon of Livestock in Jbala and \\ Ghomara (Northwestern Morocco)
}

Amina Naciri-Azzouz et Ángeles Vicente

\section{Introduction}

Dans cet article, on a essayé de saisir le savoir-faire d'une société paysanne sur l'élevage des animaux, une activité qui fait, ou faisait, partie de sa vie quotidienne et de l'économie traditionnelle. La région étudiée est située dans le nord-ouest marocain, du point de vue géographique, dont une partie est connue comme "Jbala», alors qu'une autre partie a conservé la dénomination confédérative historique de "Ghomara ${ }^{1}$. Notre propos est de nous concentrer sur le lexique spécifique de l'élevage, relatif à sa pratique et aux champs sémantiques du bétail et du labourage. 
Figure $1:$ Jbala et Ghomara

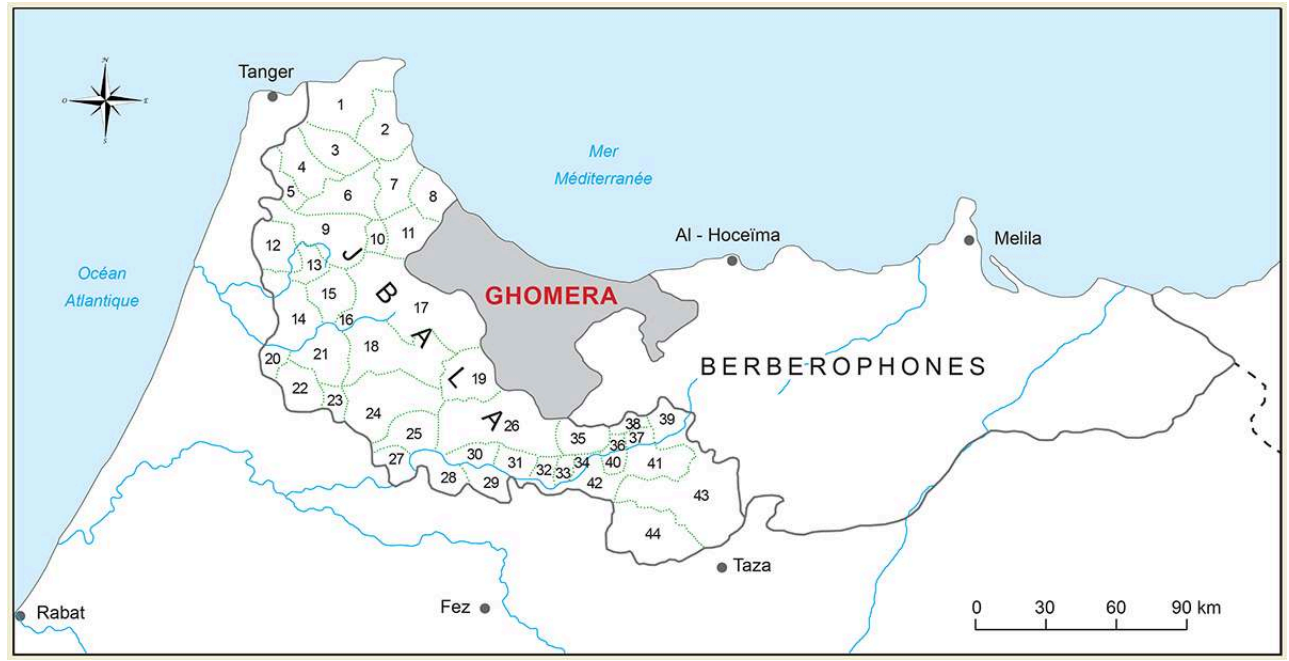

Vignet-Zunz 1995 : 2

2 Les travaux avec une approche lexicologique, dédiés aux variétés arabes ne sont pas très nombreux, surtout si on compare avec la situation d'autres langues. Ainsi, on manque d'études sur certains champs sémantiques spécifiques ou de travaux comparatifs entre les différentes variétés de l'arabe.

Bien sûr, il y a des exceptions mais elles ne sont pas nombreuses et il y a beaucoup de travail à faire dans ce domaine-là. Le travail de Peter Behnstedt \& Manfred Woidich (2011-2014) Wortatlas der arabischen Dialekte, avec trois volumes, est l'une des exceptions à cette situation.

4 Par rapport à la région du nord-ouest du Maroc, il y a quelques études linguistiques qui ont décrit et analysé principalement les traits de type phonétique, morphologique et syntaxique (Vicente et al. 2017). Mais il y a beaucoup à dire dans le domaine de la lexicologie, surtout du point de vue de la diachronie, avec l'exception des travaux de Colin sur les étymologies maghrébines (Colin 1926, 1927 et 1930).

5 Pour le lexique du bétail à Jbala et Ghomara, il y a des informations dans le domaine de la géographie dialectale, par exemple, un chapitre dédié à la faune dans le premier volume du travail de Behnstedt \& Woidich (2011-2014) cité ci-dessus, et aussi les articles de Behnstedt (2005 et 2007); en lexicographie, on trouve les acceptions recueillies dans les dictionnaires, par exemple celui de Prémare et al. (1993-1999) et dans le domaine de la dialectologie arabe, on a le travail de Heath (2002) entre autres. Pour ce qui est de la littérature orale, Westermarck a recueilli plusieurs proverbes qui font référence aux animaux (Westermarck 1930).

6 Sur les autres variétés maghrébines de l'arabe, il y a aussi quelques références à citer, voir par exemple : Cassar-Pullicino 1967 et Monteil 1951 et 1952.

7 Aussi, les berbérisants ont relevé beaucoup d'informations sur le vocabulaire des animaux dans les différentes variétés berbères avec des approches différentes, par exemple, la trace du contact linguistique dans ce lexique, la lexicologie, etc. (voir entre autres Basset 1936-1939, Kossmann 2013, l'Encyclopédie Berbère (plusieurs entrées)).

8 Les animaux sont très présents dans la société marocaine, et leur représentation apparaît dans des domaines variés, comme Goldenberg l'a montré. D’après cet auteur, 
ils sont représentés dans divers arts et artisanats traditionnels et dans la publicité, et ils sont présents également dans toutes les formes de la littérature orale (proverbes, chansons, poèmes, contes, légendes, etc.) (Goldenberg $1999: 49$ et 51).

9 Ainsi, il existe tout un lexique commun et très connu partout au Maroc qu'on ne va pas analyser dans ce travail. L'intérêt qui nous a amené à cette recherche est de relever les mots spécifiques à la région de Jbala et Ghomara ou pas très habituels dans d'autres variétés arabes marocaines.

10 Le lexique de l'élevage est en danger de disparition à cause des deux situations suivantes: d'abord, toutes les formes d'activités traditionnelles s'expriment dans la langue quotidienne, une langue qui évolue rapidement dû au processus de nivellement dialectal qui a lieu dans les variétés arabes du Maroc. Et en deuxième lieu, car les transformations socio-économiques de la région étudiée supplantent les activités traditionnelles, ce lexique est en train de tomber en désuétude avec la modernité et l'urbanisation de la population. En ce sens, on a remarqué que les plus jeunes n'effectuent plus ce type de travail, et ne connaissent pas ce lexique; ainsi il arrive qu'ils ne prêtent pas attention, le considérant comme démodé ou stigmatisant.

\section{Le terrain}

11 Les données présentées ont été recueillies à partir d'un travail de terrain collectif mené pendant un mois, en mars 2014, dans plusieurs villages et hameaux de la région des Jbala. Pour la région de Ghomara, le travail de terrain a été plus long, entre mars 2014 et décembre $2015^{2}$, car il fait partie d'une thèse doctorale sur les variétés arabes de Ghomara.

12 Nous sommes allées dans quelques villages situés à l'intérieur et dans d'autres situés sur la côte, de telle façon que nous avons vu sur place les différentes façons de la population locale de gagner leur vie avec les moyens à disposition. Il s'agit, d'un côté, de deux emplacements de la région de Jbala, deux villages dans la partie nordoccidentale, et d'un autre côté, plusieurs villages de la région de Ghomara sur la côte et à l'intérieur.

Dans le premier cas, il s'agit des douars Bni Hlou (commune rurale d'Anjra) et Ajibech (commune rurale de Saddina). Ils sont à $30 \mathrm{~km}$ (environ) de Tétouan; le village le plus proche connecté par une route est Khmis Anjra, à partir duquel, il y a $5 \mathrm{~km}$ pour Bni Hlou et $10 \mathrm{~km}$ pour Ajibech. Ils ne sont pas accessibles par une route, mais il faut utiliser des chemins sans asphalte et monter soit à pied, soit en fourgonnette ou en âne. Tous les exemples cités dans ce travail sont tirés du parler d'une femme âgée entre 60-70 ans et née à Bni Hlou, et du parler d'une femme et d'un homme nés à Ajibech, ils sont des frères, âgés entre 30-40 ans.

Dans le deuxième cas, les localisations étaient le long de la côte et à l'intérieur de Ghomara : Kaa Asraas (commune rurale de Tizgane), Chemaala (commune rurale de Bni Bouzra), Darwwannu (commune rurale d'Amtar), un hameau proche de Khmis M'diq (commune rurale de Bni Selmane) et Akumsān (commune rurale de Bab Berred). La plupart des informatrices sont des femmes entre 22 et 77 ans. De toute façon, certains hommes ont participé aux conversations en apportant un vocabulaire plus lié aux techniques agricoles. 
Figure 2 : Les villages visités à Jbala et Ghomara

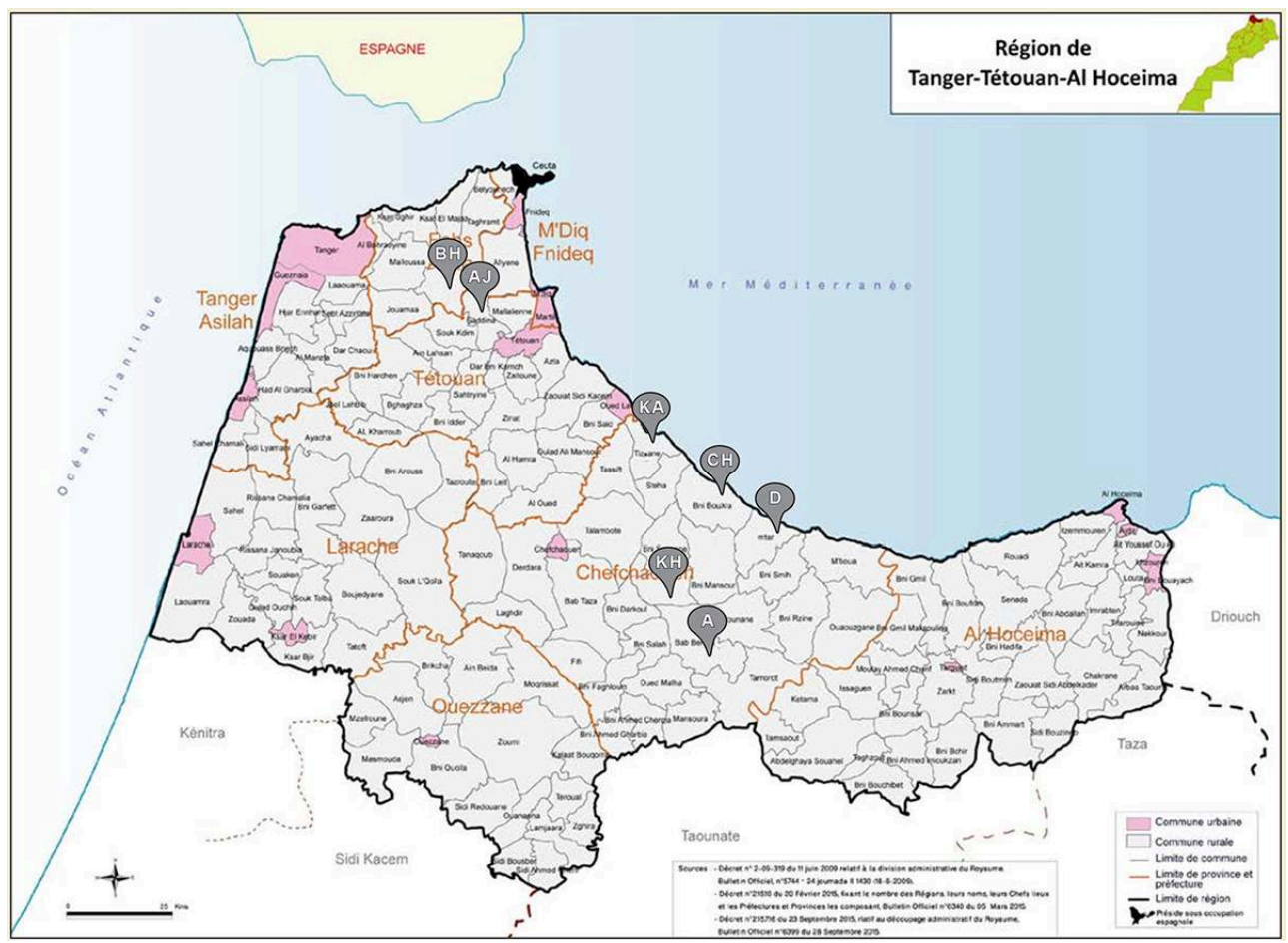

Direction Générale des Collectivités Locales 2015 : 4

Les enquêtes de terrain ont été faites par Araceli González Vázquez (anthropologue), Amina Naciri-Azzouz (linguiste) et Ángeles Vicente (linguiste) au moyen d'entretiens semi-dirigés, de l'élicitation et de l'observation participante. Ainsi, pour la récolte des données, on a posé des questions très générales sur le monde animal et la vie quotidienne des informateurs, en laissant parler les informateurs sans interruption. Il d'agit d'une méthodologie de travail partagée par la dialectologie et l'anthropologie qui nous a fourni des données très intéressantes (voir l'article de González Vázquez dans ce volume). Il s'agit d'un corpus plus fragmenté que celui recueilli à travers un questionnaire mais, en revanche, nous disposons d'un lexique contextualisé qui nous a donné accès à d'autres informations en plus des données linguistiques, comme on le verra ci-dessous.

Nous allons donc présenter la partie du corpus que nous avons considéré la plus représentative de cette région, pas toujours exclusive. Nous avons utilisé une transcription plutôt phonologique sans considérer les variantes phonétiques caractéristiques des variétés de chaque région, sauf dans les témoins de nos informateurs pour lesquels nous avons ajouté le lieu de provenance (pour des études avec ce type d'information dialectologique, voir Vicente 2000, Heath 2002, Moscoso 2003 et Vicente et al. 2017).

Nous situons chaque terme sur le plan géographique ( $\mathrm{A}=$ relevé à Anjra, $\mathrm{GhO}=$ relevé à l'ouest de Ghomara et $\mathrm{GhE}=$ relevé à l'est de Ghomara), et nous recueillons leurs différentes variantes et acceptions dans quelques dictionnaires (Lane, Dozy, DAA, DAF). À ce type d'information linguistique, on ajoute des données ethnographiques pour offrir un aperçu de l'usage de chaque terme dans leur contexte et souligner leur aspect soit régional, soit archaïsant. 


\section{L'élevage chez les Jbala et les Ghomara}

L'élevage chez les Jbala et les Ghomara est une tâche familiale destinée surtout aux femmes sauf la conduite des troupeaux à laquelle les hommes participent également (Bouillot 2010 : 92-93). Il ne s'agit donc pas de gros propriétaires et éleveurs de bétail (connus comme kəssāb). Il semble que cette activité se maintienne grâce aux femmes âgées qui rechignent à abandonner l'élevage des animaux, principalement pour la consommation domestique. Ainsi, nos informatrices ont été des femmes qui font l'élevage de petit et gros bétail pour l'économie de subsistance.

Figure 3 : Une femme žebliyya sur des terres arables (Ajibech, Tétouan)

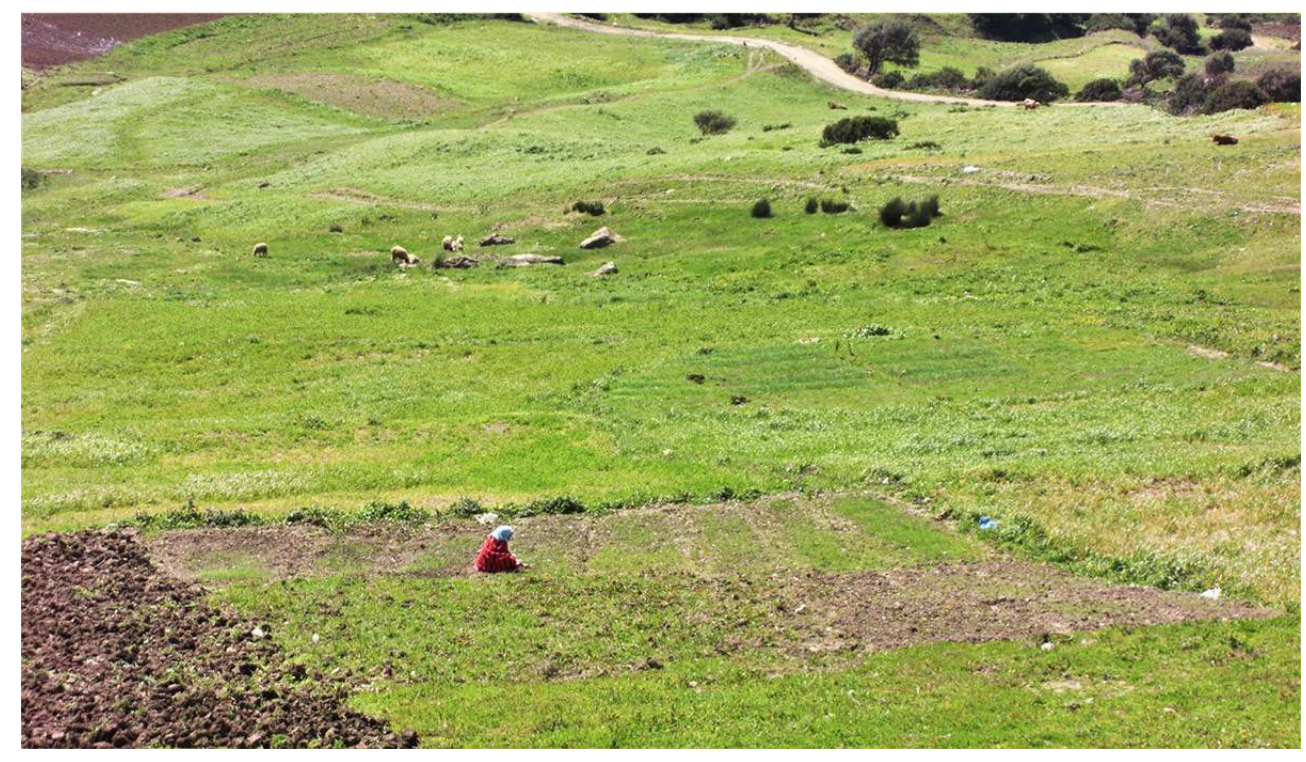

(C) A. Naciri-Azzouz et Á. Vicente comme le montre l'exemple suivant (femme de Chemaala d'environ 70 ans) :

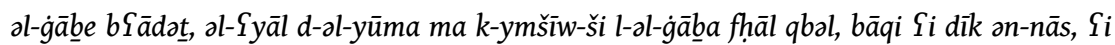
dīk am-mra lli məskīna bāqi Yānda l-mYīzāt [...] dāba ol-Yyāl xəșșa dəgles n-dāxəl șahha.

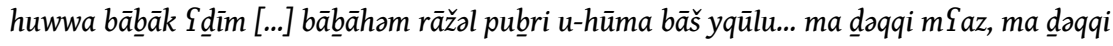
bqar, ḥna ma ntiyyḥu l-ḥzām, ngābllu t-təlfāza u-b-ṛ-rāṣ̆ $u^{3}$.

«La forêt s'est éloignée, les jeunes femmes [lit. gamins] d'aujourd'hui ne vont pas à la forêt autant qu'auparavant, seulement cette femme qui la pauvre a encore des chevrettes [...] Maintenant les jeunes femmes veulent rester dedans de force. Est-ce que ton père est faible [...] Leur père est un homme pauvre et elles pour dire... Elle n'élève ni chèvres, ni vaches, on ne perd pas l'hzom [la ceinture], on s'occupe devant la télévision et il se débrouille tout seul! ».

Les bovins et les ovins sont élevés en plaines, alors que les caprins ont une meilleure présence dans les montagnes à cause de l'orographie dans les régions montagneuses (Raki \& Haj Soulami 2001). Ainsi, on fait la différence entre ksïba rqīqa " petit bétail, ovins et caprins » (ǵnam u mYaz) et ksiba g liẹda « gros bétail, bovins » (bqar). Les paysans de la région utilisent aussi l'expression : əl-Sard əl-kbir pour le gros bétail ${ }^{4}$. 
Figure 4 : Une chèvre, deux chevrettes et un mouton mangent dans une bassine en plastique (Tizgane, Chefchaouen)

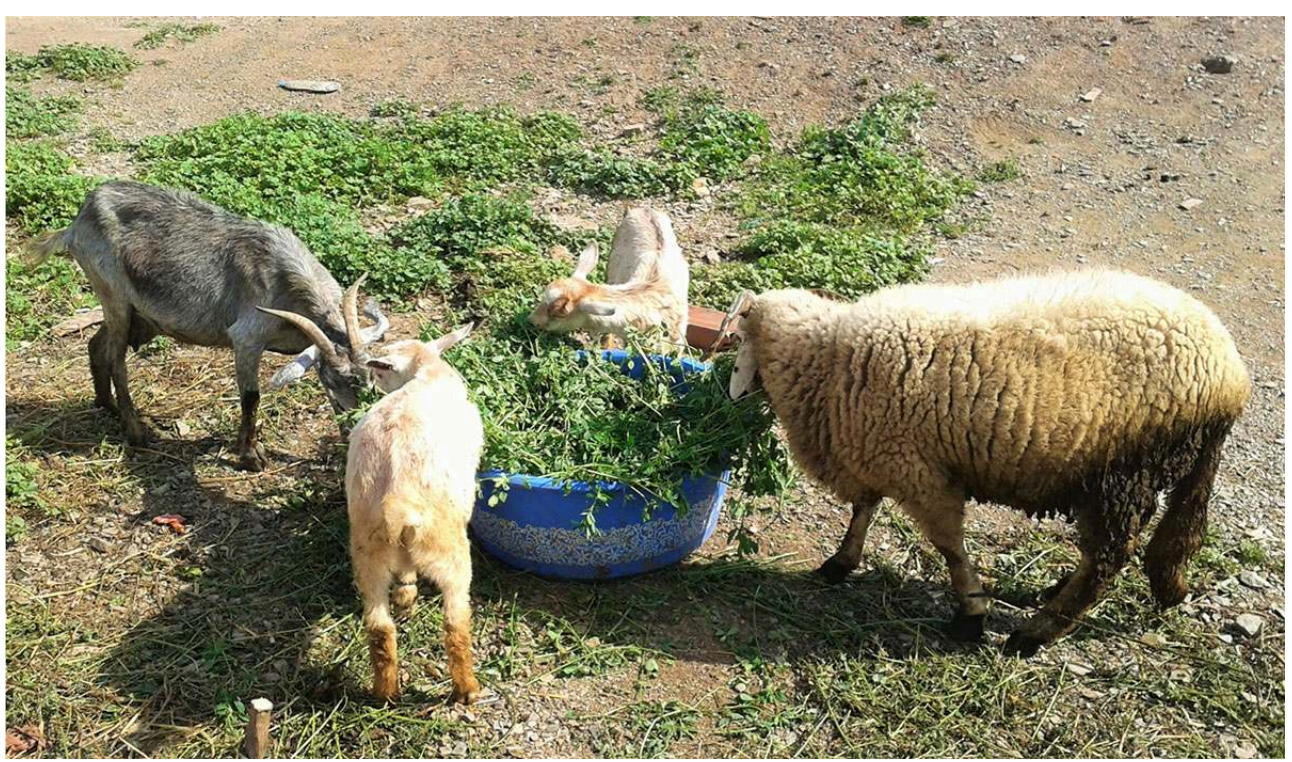

(c) A. Naciri-Azzouz et Á. Vicente

21 Le bétail est employé aussi pour labourer la terre. Les paysans utilisent bqar « vaches », bǵal «mulets", xyūla «chevaux» et ḥmìr «ânes». Bien sûr, il y a la possibilité de labourer avec des tracteurs : lli yḥ̂rtu b-əl-mwātạ « qui labourent avec les tracteurs ».

22 Cependant, l'élevage est une activité qui recule chez les Jbala. Selon les différents rapports de la $\mathrm{FAO}^{5}$ : la déforestation, le manque d'eau, la cannabiculture, etc. l'ont déjà déplacé. En tout cas, il y a des différences entre les deux terrains visités. Il semble que chez les Ghomara ce processus est plus rapide et il n'est pas facile de trouver aujourd'hui des gens qui s'occupent de l'élevage comme activité principale ${ }^{6}$, malgré les différents projets qui visaient l'amélioration et la valorisation de la production laitière aux environs de la ville de Chefchaouen. D'autre part, sur la côte de Ghomara et à Anjra, grâce à une extension plus grande de terres plates pastorales et l'achat du lait par la coopérative locale (Colainord, Coopérative laitière du nord, située à Tétouan), l'activité d'élevage est plus répandue.

23 Toutefois, comme on l'a déjà signalé, les changements socio-économiques au sein de la société jebli sont en train de remplacer ces activités par le secteur des services, la pêche, le petit commerce local, etc.

\section{Le lexique sur l'élevage chez les Jbala et les Ghomara}

Quelques noms d'animaux (la femelle et le mâle) sont des termes pan-dialectaux dans le monde arabophone. De cette façon, on les trouve aussi dans les deux régions choisies. Voir quelques exemples dans le tableau 1 : 
Tableau 1 : Noms d'animaux communs au monde arabophone

\begin{tabular}{|c|c|c|c|}
\hline & A & Gho & GhE \\
\hline Vache & baqra pl. bqar & baqra pl. bqar & baqra pl. bqar \\
\hline $\begin{array}{l}\text { Cabestro } \\
\text { Taureau }\end{array}$ & $\begin{array}{l}\text { fhal pl. fhüla } \\
\text { tawr pl. țirăn }\end{array}$ & fhal pl. fhūla & $\begin{array}{l}\text { fhal pl. fhüla } \\
\text { atawar tawar pl. tîrăn }\end{array}$ \\
\hline $\begin{array}{l}\text { Brebis } \\
\text { Bélier ou agneau }\end{array}$ & $\begin{array}{l}\text { ganma pl. gnam } \\
\text { hawli pl. hwwâla }\end{array}$ & $\begin{array}{l}\text { ganma pl. gñm } \\
\text { hawli pl. ḥwâla }\end{array}$ & $\begin{array}{l}\text { gəlma gənma } \\
\text { hawli pl. hwāla }\end{array}$ \\
\hline $\begin{array}{l}\text { Chèvre } \\
\text { Bouc ou chevreau }\end{array}$ & $\begin{array}{l}\text { maSza pl. } m \text { saz } \\
\text { ğdi pl. ğədyănn }\end{array}$ & $\begin{array}{l}\text { maSza pl. } m \text { 乌az } \\
\text { ğdi pl. ğədyān }\end{array}$ & $\begin{array}{l}\text { mesza pl. mৎəz } \\
\text { ğdi pl. ğədyânn }\end{array}$ \\
\hline
\end{tabular}

Dans les sept sections suivantes, nous allons présenter un vocabulaire représentatif et toujours vivant parmi les Jbala et les Ghomara en le regroupant de la façon suivante : la terminologie liée à l'âge de l'animal; nommer et interpeller les animaux d'élevage ; les instruments liés aux bêtes de somme; les endroits liés au bétail ; les actions liées au monde animal; la nourriture des animaux et, finalement, les parties du corps des animaux.

\section{La terminologie liée à l'âge de l'animal}

La terminologie liée à l'âge ${ }^{7}$ est en train de disparaître et il nous a été difficile de trouver une terminologie précise liée à cet aspect. Par exemple, les informateurs utilisent souvent les diminutifs pour désigner les petits des animaux : Yžiyyal ou Yžǐal "veau»; al-xriyyəf ou al-ġnima «agneau, agnelle»; ždīwad, mSīza «chevreau, chevrette", etc. Nous avons cependant rassemblé quelques termes avec un usage régressif mais qui montrent la richesse de ce vocabulaire :

nt $\bar{a} \bar{z}$ nsāǎ dim. ntiyyəž pl. ntūža (GhE) «le nouveau-né de la vache ou de la chèvre » verbe baqra natžat nasžat « la vache a accouché ».

Un terme qui s'utilise à Bab Berred et Bni Selmane mais que les autres informateurs de la côte de Ghomara n'ont pas identifié.

Sous cette acception on trouve dans Dozy (1881: II/639) ينتاج 《les petits de certains animaux ", mais en plus dans toutes les sources consultées apparaît une relation sémantique avec l'accouchement, la parturition: en arabe marocain (DAF 11:310) «produit (des troupeaux)»; en arabe andalou nitāğ « parturition » (DAA $2017: 1241$ ), ainsi que dans Lane (1863: 2761).

drū $b a(\mathrm{~A}, \mathrm{Gh})$ « une génisse ».

Un terme utilisé parmi les Ghzawa avec un sens très précis « la jeune vache de trois ans, génisse qui a atteint l'âge d'être saillie (mais ne l'a pas encore été) » (DAF $8: 180$ ). Le premier terme trouvé qui garde une relation étroite avec d̦ūba est darrāb "le taureau » (Dozy $1881:$ II/6), même en arabe andalou darrāb "taureau qui donne de la corne " (DAA 2017 : 787).

Selon Behnstedt (2007: 13), il s'agirait d'une forme passive sous la forme *fYill(a) et il a fait le lien avec Saddāb utilisé chez les Zaër pour désigner « le mâle du lièvre » et trēeba pour «la femelle du lièvre » chez les Bni Frāsən. Comme on vient de voir, la racine $\{d \mathrm{drb}\}$, très polysémique en arabe marocain, ou bien $\{\{\mathrm{db}\}$, ont un sens à la forme passive de « saillir une femelle » qu'on le trouve chez les Ghomara.

Néanmoins, drūba semblerait plutôt une forme sous le schème du participe passif $(\mathrm{m}) \operatorname{CC} \bar{v} \mathrm{C}(\mathrm{a}) \operatorname{m\partial f} \overline{\mathrm{u}} \mathrm{u}(\mathrm{a})$, avec la chute de la première syllabe, et le mot fait référence à : $l-$ 
baqra bāqa șgìịa, baqra bāqa ma waldat-ši Sumrra « la vache qui est encore petite, la vache qui n'a jamais mis bas ».

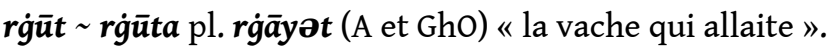

C'est un terme qu'on trouve déjà dans Lane (1863: 1112): رغُُوث " any female suckling ». Également, c'est un mot déjà recueilli par Colin chez les Ghzawa mais avec

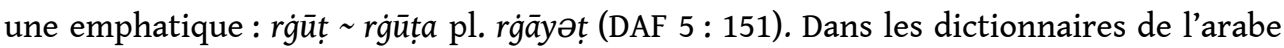
andalou (DAA $2017: 544)$ et celui de Dozy (1881: I/538) on ne trouve pas la racine \{rğt\} mais on a la racine $\{$ rǵd $\}$ avec un sens lié à l'abondance, la prospérité, etc. ou il s'agirait d'une racine sans parentage sémitique et puis elle "semble être une innovation " (selon DAA : ibid).

maggāyza pl. $\boldsymbol{m g} \bar{a} y a z \sim a m g a \bar{g} \partial z \sim \boldsymbol{m g a y z i ̄ n}(\mathrm{A}, \mathrm{Gh})$ « la vache qui ne donne pas beaucoup de lait ».

Dans Prémare et al. (DAF $10: 751$ ) on trouve différentes variantes à Rabat et parmi les Jbala, soit comme adjectifs, soit comme noms, et toutes font référence à la vache jeune qui ne donne pas de lait. Dès Ghomara au Souss, au sud du Maroc, on trouve en berbère exactement la même forme qui désigne la génisse ou bien la vache qui ne donne pas de lait :

Berbère de Ghomara (Mourigh 2016 : 391) taḡayzut - tiḡayzutatan tiḡuyaz « cow that has not given birth ».

Tarifit (Serhoual 2002 : 142) imengyez pl. imengyazen, fém. timgeyyest pl. timgeysin « veau de lait ; veau sevré ; vache laitière dont le lait commence à diminuer».

Kabyle (Dallet 1982 : 491) tameggayezt «vache, chèvre, brebis qui tarde à porter, et donne un lait appauvri »

mnīnu dim. mniwwan (GhO) « chevreau», miyyāna myāna dim. mnīwna (A et Gh) « chevrette».

mninu est un terme utilisé dans toutes les localisations, sauf à Tizgane, où il désigne le chevreau nouveau-né. Il s'agirait probablement d'une forme hypocoristique pour désigner les petits des animaux. Par exemple, le nīno (langage bébé) est un «jouet, joujou ; toute jolie chose, toute chose brillante » (DAF 11:515).

39 Pour la chevrette on a recueilli le terme l-myāna miyyāna (DAF 11:291, aussi pour l'agnelle. En berbère ghomari, tamyant pl. timyanan (Mourigh 2016: 408), et en tarifit tamiyant pl. timayanin (Serhoual 2002: 331) désigne aussi la chevrette. En berbère ghomari, il y a aussi un terme masculin amyan utilisé dans un nom composé : ayiždamyan " un chevreau sans pénis» (Mourigh 2016: 79). amyan s'utilise toujours chez les Bni Tiyyeb mais en désignant le "bouc» (Behnstedt \& Woidich 2011 : carte 91). Dans les dictionnaires arabes, on n'a trouvé aucune référence qui présente cette racine.

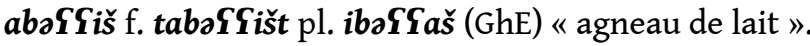

Il s'agit d'un terme relevé seulement à Amtar et Bni Selmane et inconnu par les autres informateurs, la proximité du berbère ghomari (Mourigh $2016: 103$ ), où cette forme désigne l'agneau, expliquerait sa présence. Pour l'agneau, hawli est utilisé dans ces deux localisations.

41 Une hypothèse possible pour l'étymologie d'abəS Yiš pourrait être liée au verbe onomatopéique ba؟S et bəYbəS "bêler" à partir duquel on trouve une forme d'augmentatif avec un possible suffixe latin $-\check{s}^{8}$ pour former abəS Y iš « celui qui bêle ». 
timazla « mâle et femelle de chèvre ».

Ce terme a été recueilli à Amtar en tant que collectif pour désigner les petits de la chèvre. En berbère ghomari, tamezlat pl. timezlatan c'est une chèvre qui n'a pas vêlé (Mourigh 2016: 435).

Figure 5 : Un troupeau caprin (Chemaala, Bni Bouzra)

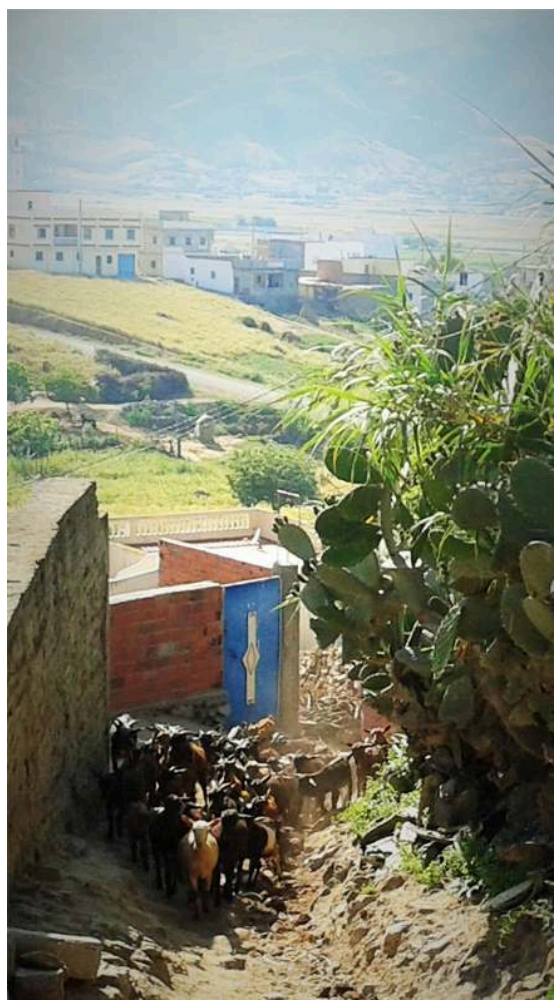

(C) A. Naciri-Azzouz et Á. Vicente

rxiyyal, raxla dim. rxila pl. rxū la $(\mathrm{GhE})$ « agneau, agnelle ».

Comme dans le cas de drūba, ce terme a un sens très spécifique chez les Ghzawa et les Zaër « agnelle, jeune brebis en âge de concevoir, mais qui n'a pas encore porté » (DAF 5 : 83). En arabe classique, on a رخلة / رخل désignant le mouton et la brebis (Lane 1863 : 1059), une forme archaïque qui désigne les adultes. À Tizgane, le terme le plus répandu est xriyyəf et les informateurs ne connaissaient pas rxiyyal. qurțāna (GhE) «poule couveuse ».

C'est un terme relevé uniquement à Bab Berred et pratiquement inconnu dans les autres localisations de Ghomara où les formes les plus répandues son qurqāna et qlūqa: d-la-dqarqar « celle qui glousse/claquète ».

Dans les différents dictionnaires arabes, les formes relevées ont une relation avec les deux derniers mots trouvés chez les Ghomara qurqāna et qlūqa, mais on n'a trouvé aucune forme relative à qurțāna (dağāăa qarqāna Dozy 1881 : II/335; en arabe andalou qalūqah < latin clŏcca DAA 2017 : 1056 ; et en arabe marocain qorqāna DAF $10: 302$ ).

On a pensé à la racine $\{q r t ̦ \sim$ qrḍ $\}$ sous la forme d'un adjectif $\{1$ u23ān(a)\} à cause de son lien sémantique avec notre terme, par exemple dans DAF (10:290), car il y a plusieurs acceptions avec un sens négatif lié à cette racine : maqrōṭ « coupé en morceaux, etc. ». Cependant, Corriente (communication personnelle, Saragosse 2017) nous a suggéré une 
possible origine latine à cause d'une contamination étymologique du cors, cortis parce que la poule quand elle couve, reste dedans et une des acceptions de corte est « une basse-cour ». Ce qui irait dans le sens de cette hypothèse c'est que dans Simonet (1888: 136) on a plusieurs formes liées à cette racine pour désigner la basse-cour. En plus, dans DAF $(10: 288)$ on trouve un emprunt qarța et sa dernière acception fait référence à « un petit champ, lopin où parcelle de terre » qui semble plutôt correspondre à l'habitat d'une poule chez les Ghomara.

\section{Nommer et interpeller les animaux d'élevage}

Donner un nom aux animaux avec lesquels les personnes ont une relation quotidienne est une pratique répandue chez les Jbala, il ne s'agit pas d'une pratique exclusive car c'est un phénomène commun à différentes cultures (Leibring 2016).

D'après le vocabulaire en usage chez nos informateurs et selon la méthodologie de la récolte de données, les noms les plus fréquents ont été ceux des femelles et des mâles en liaison avec soit le labour de la terre, soit la traite. Ainsi, dans notre corpus on a plusieurs formes pour appeler les vaches, les bœufs, les chèvres et les boucs ${ }^{9}$ et tous font référence à la couleur et la forme de ces animaux. Les surnoms pour appeler les chèvres sont plus nombreux, tout simplement parce que les chèvres présentent une grande variété de couleurs. De plus, les informateurs affirment qu'il s'agit de surnoms qui permettent de s'adresser aux animaux car ils les connaissent; il y a donc une conceptualisation culturelle qui donne forme à un continuum lié à la manière d'appeler ces animaux :

(2) ču șobhan !̣lāh, l-Y̌̌iyyəl yəxlaq, xlaq khịhal, āw ol-bqīra u-toxlaq sorwāliyya, u-nhār tomši d Sayyat Yla l-ism dyāla, tsam Sək wo-ği wo-ḍ̂ūr

«Écoute-moi, avec la grâce de Dieu, un petit bœuf est né, il est né noir, ou bien la vache qui est née sarwāliyya, le jour où tu vas l'appeler par son nom, elle t'écoute et elle vient en se retournant $"$.

En tenant compte de la morphologie des noms recueillis, il y a une différence dans les schèmes utilisés pour construire les surnoms. Pour les vaches et les bœufs, on trouve souvent un nom formé à partir d'un adjectif avec les suffixes de la forme de la nisba en arabe maghrébin : -i (masculin) et -iyya (féminin) (Marçais 1977 : 112-113).

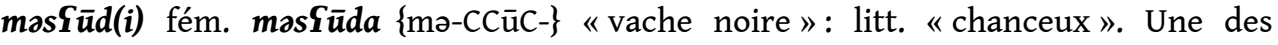
acceptions dans DAF $(6: 101)$ est « prénoms, généralement portés par des Noirs ». mərz̧ūq(i) fém. mərz̧ūqa $\{$ mə-CCūC\} « vache noire » litt. « qui a de la chance ».

wardi fém. wardiyya $\{1 \mathrm{v} 23 \mathrm{i}\}$ « vache rouge » litt. « rose ». sorwālli) fém. sorwāla sorwālya $\{1 ə 23 \bar{a} 4\}$ "avec les pattes d'une autre couleur », ou "avec des poils noirs et blancs». Il s'agit d'une allusion claire aux culottes, sorwāl en arabe marocain. En plus, dans DAF $(6: 86)$ on trouve la même appellation mais dans le cas des chevaux chez les Zaër.

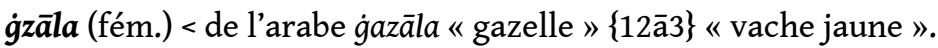

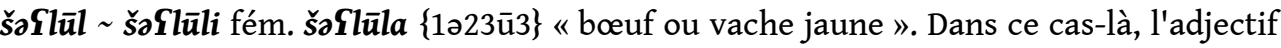
provient de la racine $\{$ ṣ̌l\} « allumer, illuminer », c'est-à-dire, ce qui est illuminé(e).

Syūniyya $\{12 \bar{u} 3-i y y a\}$ : « vache rouge et noire ». 


\section{Les instruments liés aux bêtes de somme}

on l'a déjà signalé, le bétail est utilisé pour labourer la terre à Anjra et chez les Ghomara, mais les tracteurs sont employés quand c'est possible. À l'intérieur de la région Ghomara par exemple, le bétail est plus utilisé pour labourer la terre que sur la côte :

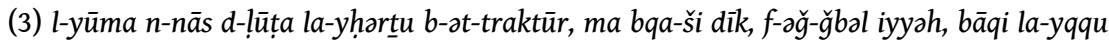
l-bhāyam, bāqi la-yqqu l-hmirr, bāqi la-yqqu b-l-bqar

«Aujourd'hui les gens de la plaine labourent avec le tracteur, on ne le fait plus comme ça. Dans la montagne oui, ils utilisent encore les bêtes de sommes pour labourer, ils utilisent toujours les ânes et les vaches ».

asadmar (GhO) azatmir (GhE) « entrave ».

Un terme recueilli à Ghomara où deux variantes ont été attestées : asədmər à Tizgane, à l'ouest et azațir à l'intérieur, à Bni Selmane. Il s'agirait d'un nom d'instrument berbère avec le préfixe berbère $s$ - et dont la racine $\{\mathrm{dmr}\}$ garde la signification en kabyle de «donner des coups ; pousser, foncer du poitrail, etc.» (Dallet 1982:144), et en berbère touareg " être attaché par la tête aux pattes de devant la vache (pendant qu'on la trait)» (Heath $2006: 83$ ).

Naiit-Zerrad (1998 : 345) a relevé le même mot au Maroc Central, en tamazight, avec la même signification: un féminin taseddmert « collier d'attache d'une bête » et iseddmer « corde qui sert à attacher le veau au pied de sa mère ». 
Figure 6 : Une entrave (Tizgane, Chefchaouen)

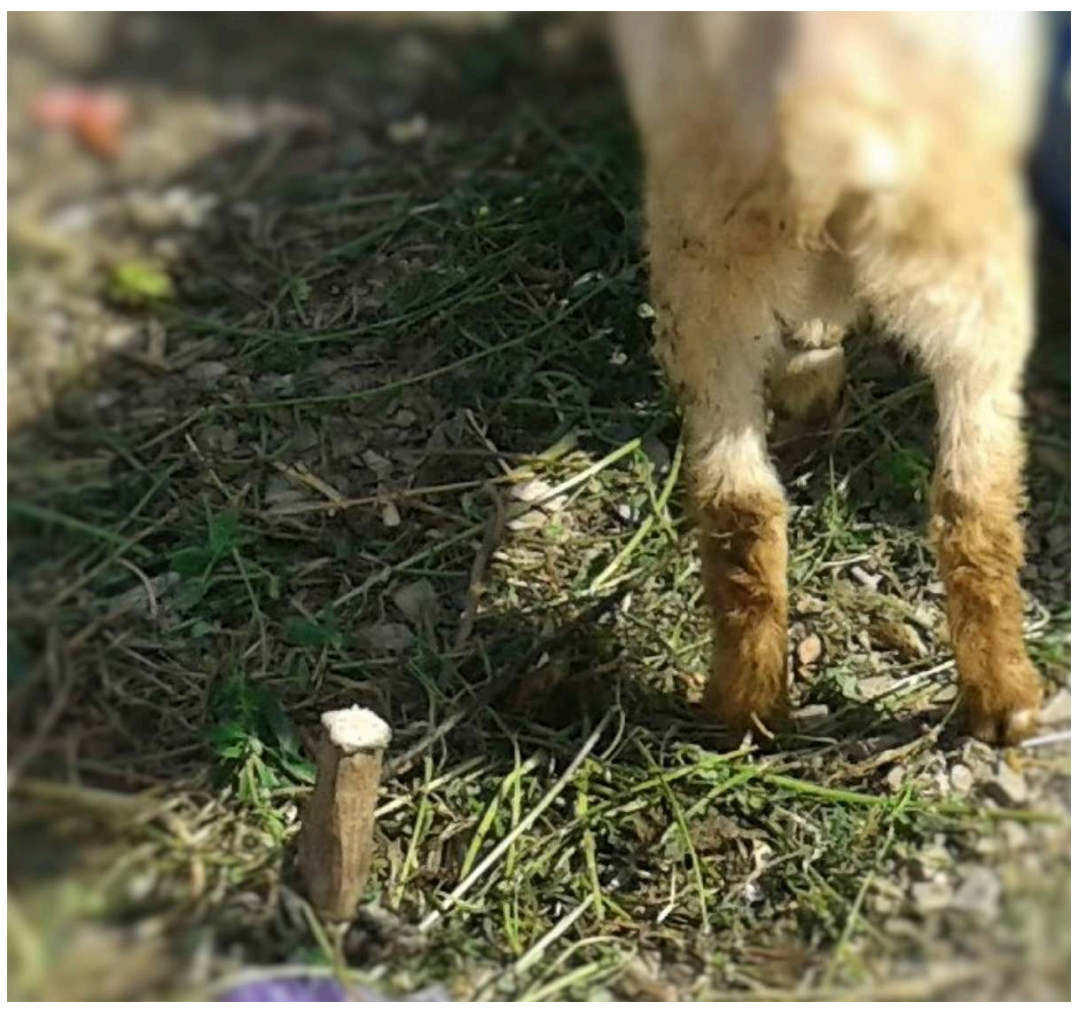

(c) A. Naciri-Azzouz et Á. Vicente

$69 x a l x \bar{a} \mathbf{l}(\mathrm{A}, \mathrm{GhO})$ « entrave » (DAF $4: 120)$ C'est un terme utilisé à Anjra et aussi parmi les Ghomara en co-variation avec asədmar.

$70 \mathbf{x u d m i}(\mathrm{GhO})$ " panier qui est utilisé pour stocker les herbes ». Dans DAF (4:30) c'est plutôt le poignard.

71 a Sallāf (GhO, GhE) « panier utilisé pour stocker les herbes pour l'élevage ». Dans ce cas, on trouve un schème de métier avec le préfixe masculin berbère $a$ -

azəg்lu (A, GhO, GhE) «joug ». C'est un terme berbère relevé dans les variétés berbères marocaines et algériennes (Mourigh 2016 : 292 ; Ibáñez 1959 : 379 ; Renisio 1932 : 325 ; Dallet 1982 : 935); et qu'on trouve toujours dans les parlers arabes des Jbala (Anjra, Ghomara, Branès (Colin 1921: 103) et jusqu'à l'Ouargha sous la forme zaglo (LéviProvençal 1922 : 196) ; ainsi qu'à Skoura (Behnstedt \& Woidich 2012 : II/carte 268).

73 Certains ont trouvé une origine latine (iugulum), mais d'autres l'ont mis en doute à cause d'une adaptation problématique du latin (Colin 1927 : 64-65, Kossmann 2013 : 63/ no12).

En berbère ghomari, on a la racine $\{(\mathrm{s}) \mathrm{gl}\}$ dans les noms suivants : asaḡul "crochet " (Mourigh $2016: 419$ ) et aḡellu awellu « charrue » (Mourigh $2016: 389$ ) et, en kabyle, on trouve une racine \{girw\} pour "se réunir » (Dallet $1982: 277)$ qui a donné « coller » en arabe marocain (DAF 9:372). Donc, il n'est pas déraisonnable de penser à un nom d'instrument sous cette racine qui renverrait autant à l'action de labourer qu'au fait d'attacher ensemble les taureaux. différentes variantes dérivées de la racine $\{$ swț répandues d'une manière générale parmi les dialectes maghrébins ainsi que parmi plusieurs dialectes orientaux (pour une 
discussion complète sur les différentes variantes de $\{s w t ̦$, voir Behnstedt \& Woidich $2012: \mathrm{II} / 269 \mathrm{a}, 269 \mathrm{~b}$ et 269 ).

aborțāq pl. abrāțoq (GhE) «un bâton pour faire avancer les animaux ». Il s'agit d'un terme qui présente une morphologie berbère au singulier masculin avec le préfixe $a$-, mais qui forme un pluriel arabe où le morphème masculin singulier berbère a été lexicalisé.

Dans DAF 1:17, on trouve à Tanger ābortāq «aiguillon de laboureur", et bortāq pl. brātaq «sorte de poinçon utilisé par les Juifs raccommodeurs de vaisselle » (DAF 1: 170). En tarifit (la variété berbère du Rif), on trouve un emprunt arabe pour désigner le même instrument bertaq pl. rbrateq "aiguillon» (Serhoual 2001-2002 : 29). Selon Colin (1927 : 57) qui l'a relevé à Anjra et Tanger, il dérivait du latin pertǐca « perche, gaule ».

78 giyyāra (GhE) « une sorte de planche (en bois) à découper ».

À Ghomara, on a deux variantes qiyyar (GhO) et giyyar (GhE) pour désigner les branches d'un arbre qui s'utilisent pour le feu, etc. Dans Dozy (1881: II/430-331), on trouve le mot قِير plespèce de bitume dont on se sert en guise de goudron » avec un lien sémantique clair qu'on trouve déjà dans Lane (1963: 2577) : قِير .

Dans le tarifit (Serhoual 2001-2002: 144), on trouve le verbe geyyer «se mettre à l'ombre ; se reposer (bête) », mais aussi agiyyur « souche, tronc, tronçon ».

mag̀māṭ (A) «masque anti-mouches ». Un nom d'instrument formé sur la racine \{ǵmḍ\}, c'est-à-dire, ce qui bande les yeux. Dans DAF $(9: 419)$, on trouve la même forme mais seulement utilisée au pluriel. Une variante ġommāda a été attestée aussi pour les Jbala par Colin (DAF $9: 418$ ).

81 məḍad pl. $\boldsymbol{m}$ ḍāməd (A) «joug ». C'est l'autre mot utilisé dans la région Jbala avec ce sens (voir ci-dessus azəóglu). Il s'agit d'un terme de la racine $\{d \mathfrak{d m d}\}$ qui correspond à différentes variantes de joug au Yémen, au sud-ouest de l'Arabie Saoudite, en Égypte, à Malte et en Algérie (Behnstetdt \& Woidich 2012: carte 268, et 332-333 pour une discussion sur les différentes variantes pour joug dans les dialectes arabes).

En ce qui concerne le vocabulaire rattaché aux chevaux, on trouve une terminologie arabe assez précise et stable en comparaison avec l'arabe classique et l'arabe andalou :

83 bərdā $\boldsymbol{S}$ pl. brāda $\boldsymbol{S}$ (A, GhO, GhE) «bât ».

Il s'agit d'un terme générique pour faire référence au bât. On le trouve déjà dans le dictionnaire de Lane (1863: 186) en référence à un tissu placé sous le siège du chameau, ou bien le bât dans le cas des animaux de somme. Ce mot se trouve aussi en arabe andalou bardạah ou bardaYah (DAA $2017: 122$ ), terme qui a donné en espagnol, catalan et portugais albarda (Corriente $2008: 60$ ). Le bât d'une manière générale se compose de parties différentes : 
Figure 7 : Un bât (Bni Hlou, Anjra)

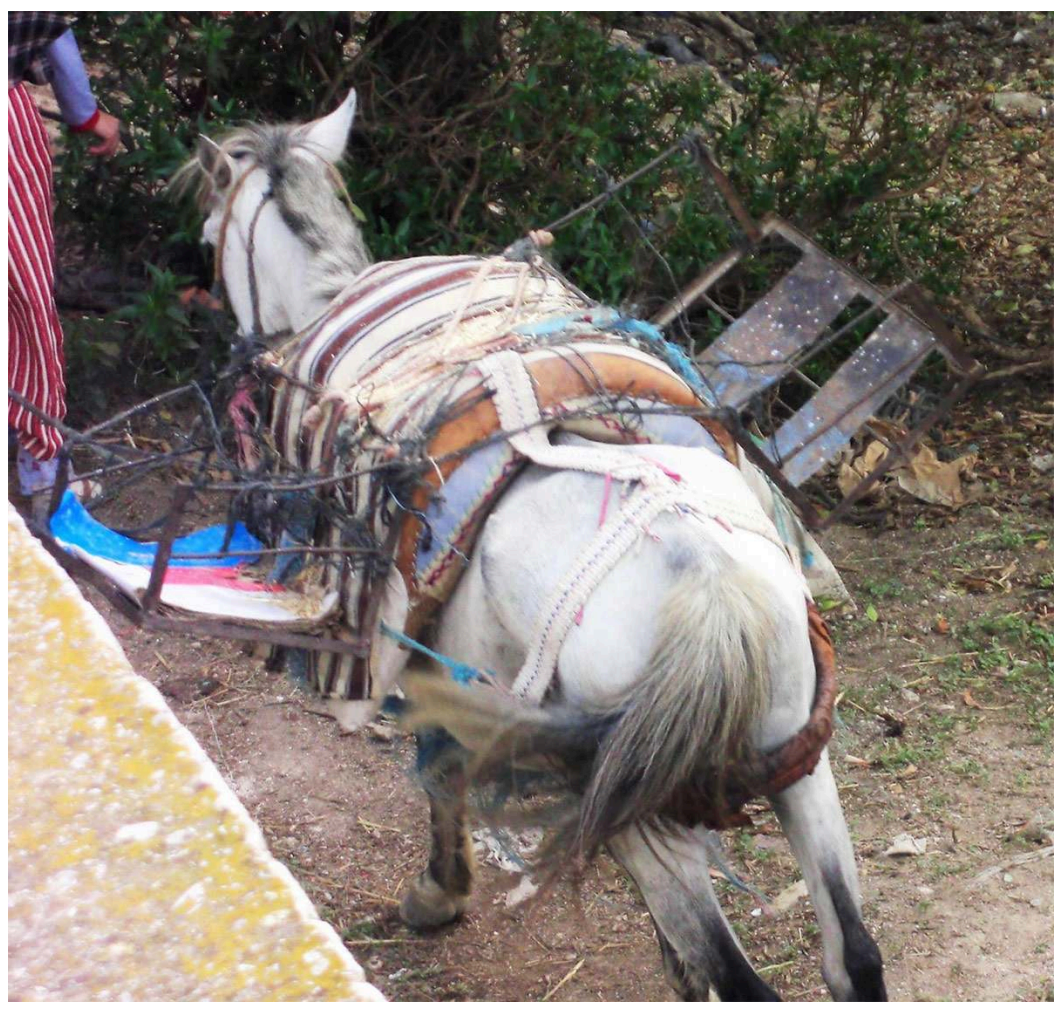

(c) A. Naciri-Azzouz et Á. Vicente

(4) l-bardāS Sanda fiha šhāl d-əs-smiyyāt [...] lli ka-ykūn məhna l-quddām ka-nqūlu lu ddayr, lli ka-ykūn fūq f-țahra morbūt f-ol-bordā S, hādāk or-roffāda. āxur lli ka-ykūn Sanda $m$-ol-ḷāwr dyāla ka-nqūlu lu tfar.

«Le bât a plusieurs noms [...] celui qui est ici en avant, on l'appelle d-dayr ; celui qui se trouve sur son dos attaché au bât, celui-là c'est or-roffāda. L'autre qui est en arrière, on l'appelle «tfar ».

dayr (A) « la partie de devant du bât ».

Dans DAF ( $4: 403)$ on trouve différentes acceptions chez les Zaër rattachées à la longe ou au poitrail des chevaux; et dans Lane (1863: 931) il y a différentes acceptions en relation avec la partie de devant et en référence à tout ce qui est rond.

rəffāda (A) « bât qui est mis sur le dos de l'animal ».

Il s'agit d'un schème d'un nom d'instrument formé sur la racine $\{$ rfd $\}$ pour «lever, charger et transporter ».

tfar (A) «trousse-queue ».

Dans Lane (1863 : 340) on a تَفَر avec exactement la même signification «the [crupper, or] strap, or thony, at the hinder part of the saddle ; [...] the appendage of the saddle that is put beneath the tailof a beast... "; ainsi qu'en arabe andalou (DAA $1997: 79,84$ ) tafaru, tafar « trousse-queue ».

87 lžām pl. ľžāmāt (A) « bride ».

Un terme arabe لِجام qu'on trouve dans Lane (1863: 3008) et en arabe andalou (DAA 2017 : 1142) liğām "bride, mors »'. Corriente (ibid) propose une origine néo-persane legam.

88 șfịha pl. șfāyəh (A) « fer à cheval ».

Dans Lane $(1881: 1369)$ on trouve quelques formes de la racine \{sfh\} qui gardent une 
relation avec les pieds, la base, etc. En arabe andalou (DAA 2017 : 765) șafihah (avec le / ș/) est « le fer à cheval ». Le même terme a été déjà relevé parmi les Jbala (DAF $8: 69)$ et aussi en berbère ghomari (Mourigh $2016: 423$ ).

\section{Les endroits liés au bétail}

gurna, gurna d-ed-dbiha $(\mathrm{A}, \mathrm{Gh})$ « abattoir ».

Dans les deux hameaux de la partie nord-occidentale de la région Jbala, où nous avons fait les enquêtes, les gens vont souvent les jeudis au village voisin plus grand, le siège du marché hebdomadaire (Khmis Anjra), pour acheter de la viande au souk. Ainsi, le bétail est seulement égorgé dans la gurna dans le cas des fêtes religieuses ou familiales. Aussi, en cas d'urgence, par exemple, si l'animal a eu un accident avec des conséquences sérieuses.

Dozy relève un terme similaire ğurn « pierre creuse, auge, bassin » (Dozy $1881:$ I/189), et aussi (Lane 1863 : 414), جُرن "hollowed stone, stone basin».

Le mot est connu sous des variantes différentes dans quelques variétés orientales et maghrébines de l'arabe : ğurn, gurn, gurna, ğarīn, yarīn, mağran, miğrān, mižarana, gannūr. Il s'agit d'un emprunt à l'araméen rabbinique (< goren « aire ; cercle, rencontre », DAA 2017 : 262). Ainsi, il a été relevé en Égypte, en Oman, en Arabie Saoudite, au Soudan, au Tchad, au Yémen et au Maroc avec le même sens « aire " (Behnstedt \& Woidich 2012 : carte $273,355-358$ ). Il pourrait s'agir donc d'un espace ouvert dans un village, où on peut situer un abattoir.

Behnstedt a trouvé au Nord du Yémen la variante gurneh « coin » (1992 : 991).

$\mathrm{Au}$ Maroc, dans Prémare et al. on trouve une autre acception "emplacement réservé aux bouchers sur un marché rural » (DAF 10 : 715), avec un lien sémantique évident.

Pour ce qui est de la prononciation occlusive, il s'agit probablement d'une dissimilation $/ \check{g} />[g]$ même si on ne trouve pas de sifflantes subséquentes ${ }^{11}$. Il s'agit d'un phénomène courant en arabe andalou (Corriente et al. 2015 : 55). Il pourrait également s'agir d'un mot très ancien arrivé d'Orient qui aurait conservé la prononciation occlusive.

a forme la plus connue pour l'abattoir dans les variétés arabes du Maroc est l'emprunt au français batwār.

domna (GhE) « un grand champ où les animaux sont égorgés, abattoir ».

On l'a relevé aussi pour Dozy et en arabe andalou avec le sens "pièce de terre labourable» (Dozy $1881:$ I/462 et DAA $2017:$ 478-479).

Dans Prémare et al. (DAF $4: 340$ ) on le trouve comme " petit jardin devant une maison ».

Il semble qu'il s'agit donc d'un champ avec des usages différents selon la région. Pour une discussion ethnographique sur le terme, voir Vignet-Zunz (2014 : 309-318).

rwa pl. rwìya (A, GhO) « étable».

L'étable est une pièce qui fait partie des demeures dans les constructions connues comme architecture rurale en terre, architecture populaire ou vernaculaire, « réalisées en terre crue comme matériau principal de structure» (González Sancho $2010: 12$ ). Lorsque la demeure a deux étages, grâce à la pente naturelle du terrain, le rez-dechaussée est utilisé comme étable pour garder le bétail. S'il n'y a qu'un étage, l'étable 
s'ouvre sur la cour ou sur le porche d'entrée. Dans les deux cas, il s'agit donc d'un système naturel de chauffage pour l'hiver.

Figure 8 : Une maison traditionnelle utilisée comme étable (Bni Selmane, Chefchaouen)

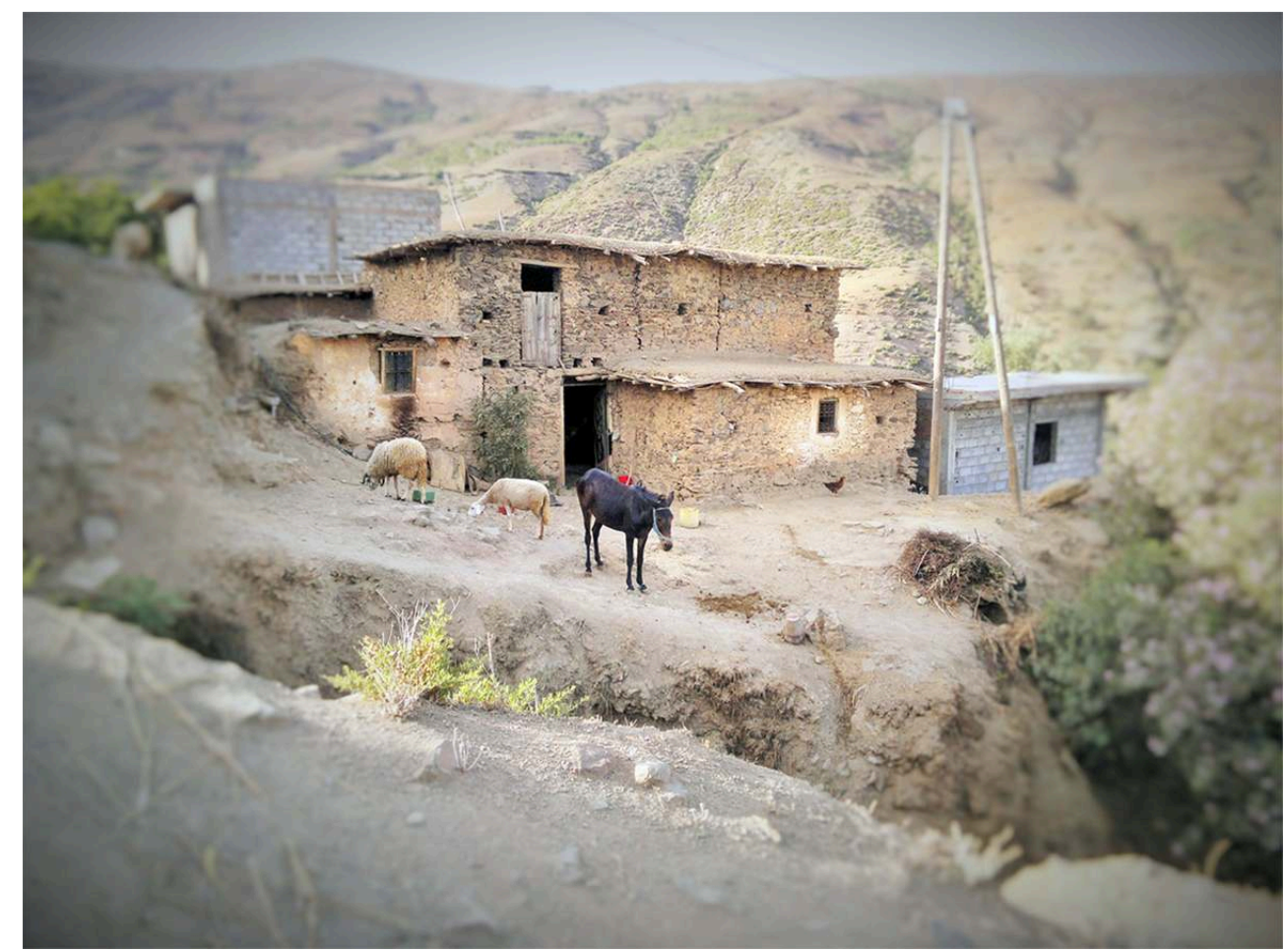

(c) A. Naciri-Azzouz et Á. Vicente

100 Cependant, aujourd'hui, il est très fréquent de trouver des familles qui habitent dans une maison auto-construite avec une structure en béton armé et des murs en brique cuite, et la construction en terre est utilisée comme étable pour garder le bétail (ibid: $46-47)^{12}$.

101 Il s'agit d'un mot relevé au Maroc, en Algérie, en Tunisie et en arabe andalou avec le même sens (Behnstedt \& Woidich 2012: carte 276, 372-373, DAA $2017: 570$ et DAF 5 : 255). Dans Lane (1863: 51), on relève déjà une forme آرِيَّة pour désigner la place où les bêtes sont confinés ; et Dozy (1881: I/574) donne les formes رِوراء et comme propres respectivement de l'arabe andalou (Espagne, sic.) et les variétés maghrébines (Afrique, sic.). 
Figure 9 : Une étable moderne avec des mangeoires (Bni Hlou, Anjra)

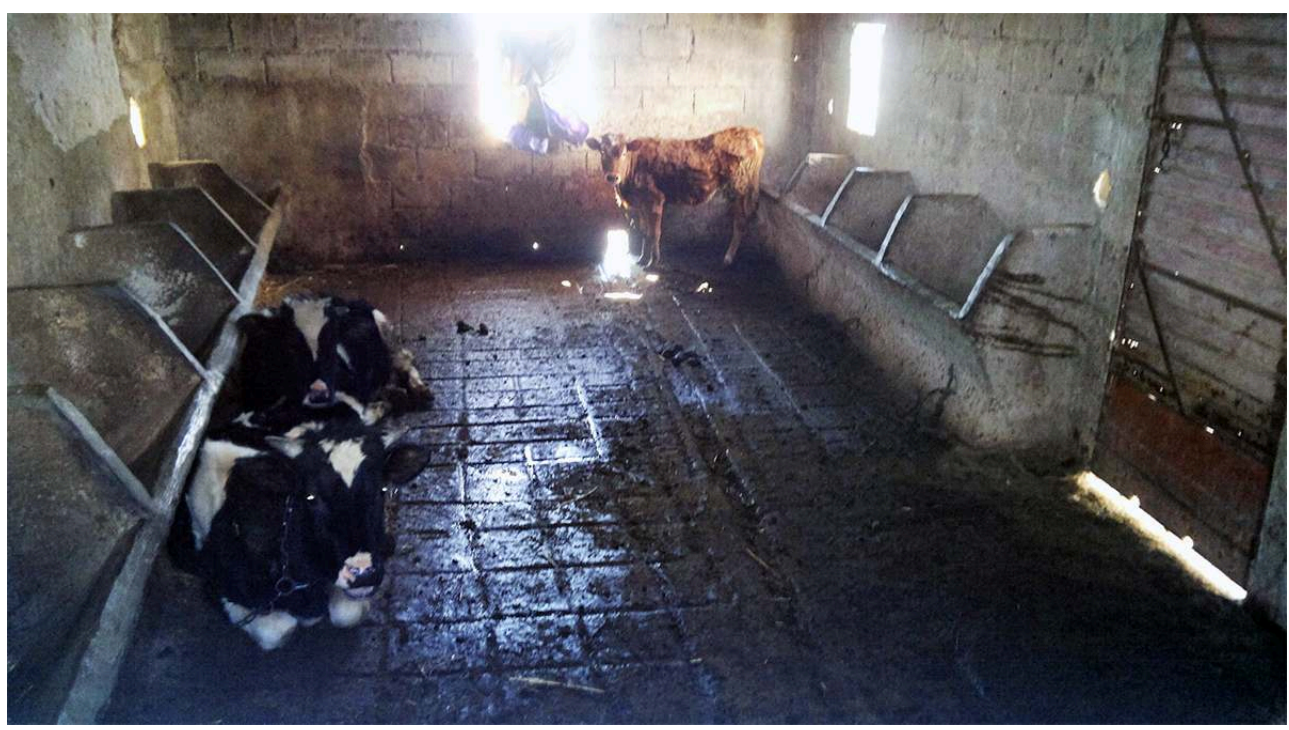

(c) A. Naciri-Azzouz et Á. Vicente

mədwād pl. $\boldsymbol{m} d \bar{a} w \boldsymbol{w} d(\mathrm{~A}, \mathrm{Gh})$ « mangeoire».

Dans Prémare et al. (DAF $4: 370$ ) on le trouve avec le même sens que nous en Jbala. En arabe andalou, madwad c'est aussi la mangeoire (DAA 2017 : 504). Lane avait déjà trouvé un rapport de ce terme avec le repas : مُدَوَّد « corn or food having in it worms and becoming eaten thereby » (Lane $1863:$ 930).

məzdḩəm d-əl-bqar (GhE) « l'étable où ils gardent les vaches et les autres animaux ».

Il s'agit d'un nom de lieu formé sur la racine $\{z h ̣ m\}$, de la VIIIème forme, "serrer ", avec le sens "lieu où se serre les vaches", un type de nom quasi inexistant en arabe marocain, et pas relevé non plus dans les dictionnaires consultés. En arabe andalou, on trouve le verbe nazdaham...muzdahim maS «se presser en foule, se bousculer » (DAA $2017: 579$ ), aussi dans les autres dictionnaires (Dozy 1881: I/582, Lane 1863: 1221 et DAF $5: 287)$.

bit d-at-tban (GhO) « grenier ».

tibn "paille " est un mot pan-sémitique relevé dans tous les dictionnaires consultés

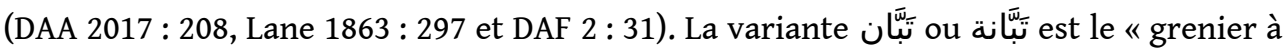
foin » (Dozy 1881: I/141) ou le « place of [or for] تِبن , (Lane, ibid).

Selon Colin, chez les Ghzawa (aussi en Jbala), on relève aussi le mot tabbāna «hutte pour abriter la paille» (DAF $2: 31$ ).

106 bərmil (A, Gh) «abreuvoir ».

Il s'agit plutôt d'un objet quotidien utilisé aussi pour abreuver les animaux chez les Jbala et les Ghomara.

107 Ce mot existe aussi dans d'autres variétés marocaines avec le sens «baril, tonneau » (DAF $1: 209$ ). D'origine bas-latine (barriculum), il est relevé aussi en andalou (DAA 2017 : 135).

$108 k \bar{a} \boldsymbol{f}(\mathrm{GhO})$ « grotte utilisée comme grenier collectif ».

Le sens le plus habituel de ce terme au Maroc est «trou, grotte » (DAF $10: 503)$, aussi en andalou, avec la variante phonétique kahf (DAA 2017 : 1125). Le mot n'a pas été relevé par Dozy ni par Lane. 
Cependant, dans les douars ou dčūr (selon la terminologie locale) montagnards chez les Ghomara, les grottes proches des demeures sont utilisées comme greniers collectifs dont se sert tout le dčar (village).

L'emploi des greniers à usage collectif est connu au sud du Maroc, surtout dans le Haut Atlas et dans la région d'Ouarzazate, où ce sont de constructions fortifiées par une muraille nommées igherm (Zarguef 2001).

\section{Les actions liées au monde animal}

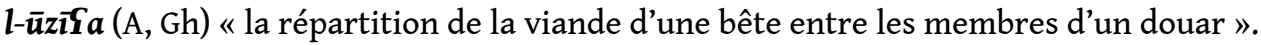
Dans les villages et hameaux Jbala et Ghomara, la viande de l'animal sacrifié est distribuée entre les gens, pour une fête religieuse comme le mawlūd ou l-Sìd oṣ-ṣgìị. Ils font la répartition de la viande en tant qu'élément rattaché à la religion.

112 Prémare et al. le définit comme « répartition et distribution de la viande d'une bête de boucherie achetée en commun » (DAF $12: 191)$. Selon Dozy (1881: II/799), cette racine a le sens de « partager » et en arabe andalou, de « distribuer » (DAA 2017 : 1344).

113 gur $\boldsymbol{\xi} \boldsymbol{a}$ et le verbe $\boldsymbol{n g a r} \boldsymbol{Y} \mathbf{u}(\mathrm{GhE})$ « répartition de la viande ».

Il s'agit de la même tradition mentionnée ci-dessus. Dans le village de Bab Berred, les hommes mettent de l'argent en commun le 26 Ramadan pour acheter de la viande et on la mange après la prière. On a relevé aussi à GhE le verbe ytfärḍu, il s'agit d'une action rattachée à la précédente car il a le sens de « contribuer à une dépense commune en versant chacun une quote-part » (DAF $10: 69$ ).

114 Dans d'autres régions marocaines, qur $\{a /$ gur $\{a$ est un tirage au sort, où la connotation religieuse semble être perdue. D'après Prémare et al., ce sont des «tas de parts de viande pour une répartition par tirage au sort » (DAF 10:300). Le même terme qurCa, avec la réalisation sourde, existait en andalou nadrab al-qurfa "tirer au sort » (DAA $2017: 1021)$.

115 Dans le lexique rattaché à la grossesse et à l'accouchement des animaux ${ }^{13}$, il y a également des termes intéressants :

116 Le verbe dxallos (Gh) « elle expulse le placenta, la délivrance ».

Utilisé aussi pour les femmes, il s'agit d'un verbe habituel en arabe marocain, trouvé dans de Prémare et al. (DAF $4: 122$ ), et en andalou (DAA $2017: 427)$ ). Par contre, Dozy (1881: I/391) ne recueille pas cette acception, mais la racine a toujours le sens de «achever, délivrer, payer». xḹs « placenta " (un terme panarabe) a une connotation positive, comme une espèce de récompense. Une informatrice de Ghomara nous a raconté qu'elles donnent du zrāS maqli «blé grillé » pour aider à la parturiente à expulser le placenta.

117 dozhom (Gh) « pousser pendant les contractions de grossesse ».

La même acception a été relevée dans DAF $(5: 287)$. En arabe andalou, ce sens n'existe mais il y a un lien sémantique avec zahánt (avec une erreur de graphie au lieu de zahámt) «presser en foule, bousculer » (DAA 2017 : 578-579); et aussi dans Lane (1863 : 1221) « he pushed him or repelled him ».

$118 d k a s s a b(G h E)$ «être fécondée ».

Le verbe est noté dans Prémare et al. (DAF 10 : 574) mais avec un autre sens et on ne le trouve pas en arabe andalou non plus (DAA 2017: 1104). Son sens est "acquérir, gagner ", un verbe de la $1^{\text {ère }}$ forme; de telle façon que la valeur factitive, qui 
correspond à la $2^{\text {ème }}$ forme en arabe, peut expliquer le sens de cette variante sémantique.

119 dtuwwah (Gh) « mugir, spécialement quand la vache est en chaleur ».

Il s'agit d'un verbe de la $2^{\text {ème }}$ forme de la racine $\{t$ twh $\}$ dont le sens trouvé dans les différents dictionnaires a un lien sémantique avec « mugir » dans ce sens basique, c'està-dire, propager un son (Lane 1863 : 1889-1990). Dans Dozy (1881 : II/66), en outre, il y a des usages proches de ceux relevés ici : طُطوَّحات الصوت «chansons que chantent les femmes chez les Bédouins [...]». Dans DAF (8: 36), la première acception de towwaḥ est "se lamenter bruyamment, brailler, etc. », mais « meugler, mugir » sont inclus également.

dəYšar (Gh) « devenir pleine, concevoir (pour les animaux)».

Trouvé aussi dans Prémare et al. (DAF $9: 112)$, ce verbe, dont la racine est liée au chiffre 10 , est utilisé avec des animaux dont les femelles ont une période de gestation de dix mois (chamelle, vache, jument). Cette acception n'existe pas en andalou (DAA 2017 : 866). Par contre, Dozy (1881: II/129) a recueilli la $2^{\text {ème }}$ forme avec le sens « accoupler, apparier pour la génération, couvrir sa femelle », ainsi que Lane (1863: 2050) عشّرت « she completed the tenth month of her pregnancy ».

\section{La nourriture des animaux}

121 D'après Bouillot (2010 : 93), l'alimentation des ruminants est assurée via des ressources pastorales relativement abondantes, trouvées surtout dans les parcours forestiers. Par contre, les reliefs escarpés sont un obstacle pour la production de fourrage. On présente ci-dessous quelques termes en rapport avec la nourriture des animaux.

Figure 10 : Des mangeoires (Tizgane, Chefchaouen)

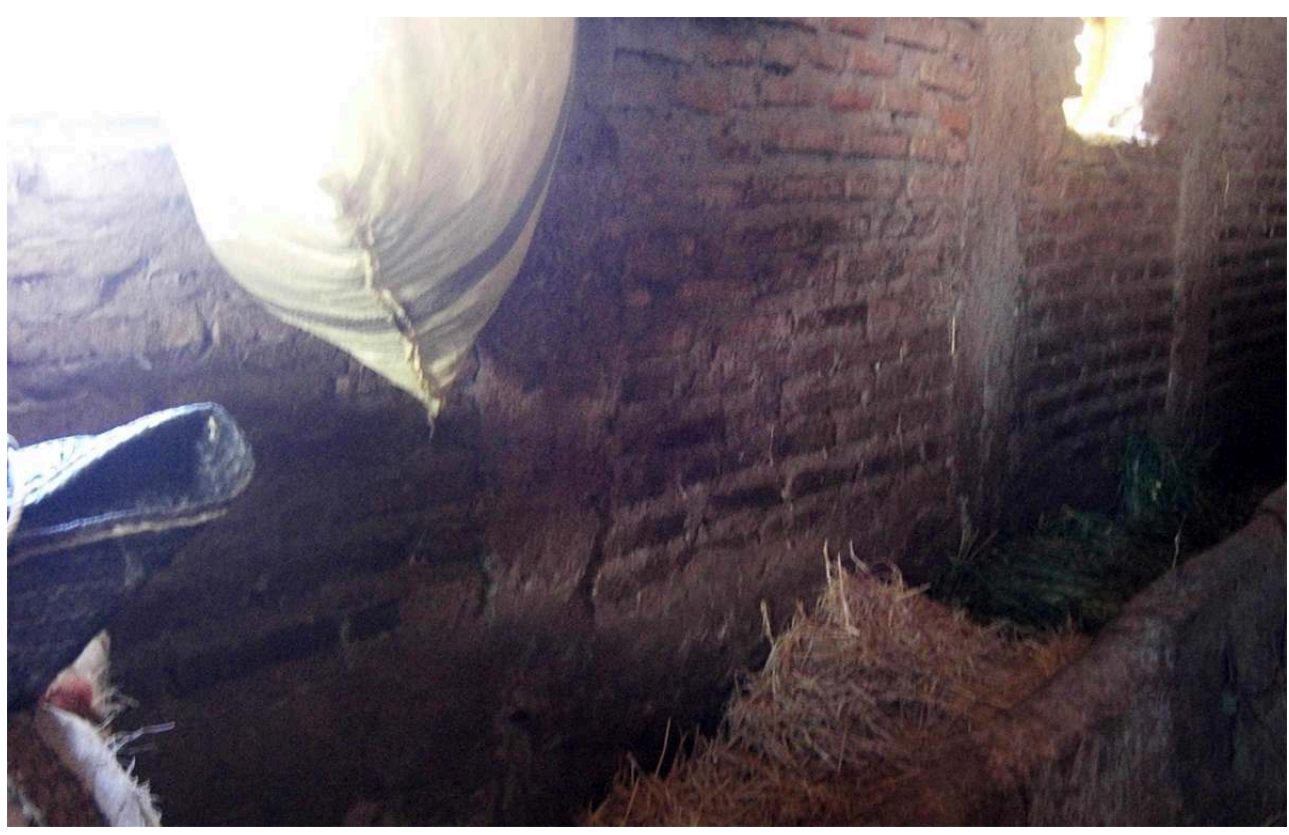

(c) A. Naciri-Azzouz et Á. Vicente

t-turkiyya (A, Gh) « maïs ».

Selon Prémare et al., il s'agit du «maïs rouge, blé de Turquie » (DAF 2:48). c'est un 
adjectif de nisba, probablement d'utilisation récente. Le mot pour le maïs dans d'autres variétés arabes marocaines est d̦ra, mais chez les Jbala ce terme signifie "sorgho " (Vicente $2000: 231$ et Lévi-Provençal $1922: 227$ ).

š-šmandar (Gh) « betterave à sucre ».

Ce terme n'a été relevé que dans DAF $(7: 191)$. Pas en andalou, ni dans les autres dictionnaires consultés.

n-nuxxāl (GhO) « semoule du blé, son ».

Colin l'a relevé chez les Ghzawa avec le sens «gros son (résultant de la mouture de céréales)»(DAF $11: 330)$.

Il s'agit d'un mot relevé aussi en arabe andalou, avec la variante nuHāl " pain de son " (DAA 2017 : 1250), aussi نخال dans (Dozy 1881 II : 650) ; la même racine \{nxl\} exprime le verbe « tamiser » et « le tamis ». Selon les auteurs, il s'agit d'une racine pan-sémitique.

l-fașșa (GhO) « luzerne ».

Ce mot a été recueilli déjà par Kazimirski (1875: III/759) fasfașa, avec le sens « espèce de plante qui donne le meilleur fourrage ». Il a été relevé avec la même forme en arabe andalou, il s'agit d'un emprunt au pehlevi à travers l'araméen (DAA 2017: 970). Au Maroc, on le trouve dans Prémare et al. (DAF $10: 110)$.

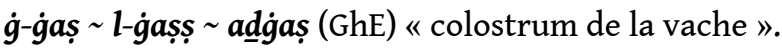

C'est un berbérisme commun au nord du Maroc et documenté aussi en arabe andalou (Ferrando 1997: 110; DAA 1997: 180). Il s'agit d'un terme qu'on trouve depuis le berbère ghomari ad yeș (Mourigh $2016: 380$ ) jusqu'au touareg adayas (Heath $2006: 74$ ).

\section{Les parties du corps des animaux}

129 Les noms des parties du corps du bétail coïncident largement avec ceux utilisés pour le corps de l'être humain. Ainsi, il existe, par exemple, rāa « tête ", fwād « entrailles ", qalb « cœur », ržilāt « jambes, pattes », karš « ventre », etc. Voir Behnstedt \& Woidich (2011).

Par contre, quelques termes sont utilisés seulement dans des contextes précis, toujours rattachés à ces animaux. On fera référence dans ce paragraphe à des termes qui illustrent certains aspects de la vie quotidienne dans cette société montagnarde.

131 mașrāan pl. mșārạn (A, Gh) «boyau, intestin ».

Les intestins et les tripes en général font partie de la gastronomie marocaine où l'on trouve plusieurs plats (Abu-Shams 2002). Pour la cuisine, seulement l'intestin grêle est utilisé :

(5) māši l-mșāạan kāmlīn, fiham dik or-rqīwqīn, hādūk la-nqalbūhom b-əl-Sawd u nanțafraham. ol-mașrān al-g̈lat la-ysiyybūham.

«On ne mange pas tous les intestins, il y a ceux qui sont fins [intestin grêle], cela on

les retourne avec l'aide d'un bâton et on les tresse. Le gros intestin, ils les jettent ».

Chez les Ghomara, il existe une technique particulière, appelée $\mathbf{l}-\mathbf{k r a \overline { d } d a l}$ (pl.) « saucisse à base de panse et de tripes séchés au soleil ». La même technique a été également relevée par Colin « sorte de saucisse de foie enveloppée de crépine, mise à sécher et que l'on met en chapelet, pour brochettes » (DAF 10:546); et par Abu-Shams (2002: 277) kūrdāla « especie de embutido de tripas picadas [...]».

Pour l'étymologie de krādal, différentes entrées de l'arabe andalou dans Dozy (1881: II/ $326,454)$ semblent avoir une relation sémantique avec krādal: 

dans différentes variétés berbères sous la même forme: berbère de Ghomara amzellaf 
«sheep's head» (Mourigh $2016:$ 435), berbère de Senhaja azallif «tête » (Renisio 1932 : 143) ou " tête de sanglier » (Ibáñez $1959:$ 84), et en tarifit azeğif « tête » (Serhoual 2002 : 674) , parmi d'autres.

Il s'agit d'un mot hybride avec une racine berbère, combinée à un schème nominal arabe marocain $b u$ (père) + un attribut, voir une construction semblable bu-g்ummāsi dans l'article Caubet \& Aumeeruddy-Thomas, dans ce volume).

\section{Conclusion} origine arabe. Parmi les 51 mots étudiés ici, sans compter les surnoms et variantes, seuls 10 ont une origine possiblement berbère ou latine/romane. Il s'agit d'un lexique archaïque, utilisé dans des contextes dans une certaine mesure conservateurs et liés à la vie traditionnelle. Par exemple, la terminologie relevée à Anjra en référence aux chevaux ( $t$ far et dayr), a été trouvée dans Lane pour ce qui concerne les chameaux dans certains cas. 
Les mots d'origine berbère sont peu nombreux et clairsemés dans les différents champs de spécialité (maggayza, timazla, asadmar, azaglo et azellif). Cependant, la présence du berbère se trouve en particulier dans la morphologie, et surtout chez les Ghomara, où on trouve le préfixe masculin berbère $a$-, même en pluriel.

En ce qui concerne les mots avec une possible origine latine/romane (qurțāna, aborțaq, bormill, krādal et frāqaš), les analyses pour l'instant ne permettent pas d'aller plus loin.

Cet article montre cependant la richesse du vocabulaire de l'élevage, en même temps qu'il témoigne d'une certaine variation, il montre une homogénéité dans la région Jbala et Ghomara, et ceci, bien que nous soyons dans une région montagnarde où les liens entre eux ne sont fréquents ni toujours faciles.

\section{BIBLIOGRAPHIE}

Abu-Shams L. 2002 - Estudio lingüístico y textual del léxico relativo a la cocina y la alimentación en el dialecto árabe de Rabat (Marruecos). Zaragoza, Universidad de Zaragoza.

Badawi E-S \& Hinds M. 1986 - A Dictionary of Egyptian Arabic. Arabic-English. Beirut, Librairie du Liban.

Basset A. 1936 - Atlas linguistiques des parlers berbères, Algérie-Territoires du Nord. Noms d'animaux domestiques. 1. Cheval, mulet, âne. Algiers, Université d'Alger.

Basset A. 1939 - Atlas linguistiques des parlers berbères, Algérie-Territoires du Nord. Noms d'animaux domestiques. 2. Bovins. Algiers, Université d'Alger.

Behnstedt P. \& Woidich M. 2011 - Wortatlas der arabischen Dialekte. Band I: Mensch, Natur, Fauna und Flora. Leiden-Boston, Brill.

Behnstedt P. \& Woidich M. 2012 - Wortatlas der arabischen Dialekte. Band II: Materielle Kultur. Leiden-Boston, Brill.

Behnstedt P. \& Woidich M. 2014 - Wortatlas der arabischen Dialekte. Band III: Verben, Adjektive, Zeit und Zahlen. Leiden-Boston, Brill.

Behnstedt P. 1992-2006 - Die nordjemenitischen Dialekte. Teil 2: Glossar. 3 vols. Wiesbaden, Dr. Ludwig Reichert.

Behnstedt P. 2005 - Materialien für einen Dialektatlas von Nordost-Marokko I: tiernamen, teil 1: Einleitung, Transkription, Ortsnamenverzeichnis, Karten. Revista de estudios de dialectología norteafricana y andalusí $9:$ 7-72.

Behnstedt P. 2007 - Materialien für einen Dialektatlas von Nordost-Marokko I: tiernamen, teil 2: Kommentare, Bibliographie II: Mensch: Körperteile, Tei1 1: Karten, Teil 2: Kommentare, Revista de estudios de dialectología norteafricana y andalusí 11 : 7-57.

Bouillot A. 2010 - Éleveurs de montagne. In Bouchmal F. Calvo Valderrama G.-I., Jabiot I. \& Vignet-Zunz J. (Coord.), Regards sur les patrimoines et les terroirs des Jbala. Chefchaouen, Ministère de la Culture : 92-97. 
Camps G. \& Vignet-Zunz J. 1998 - Ghomâra. Encyclopédie berbère XX. [En ligne] URL : http:// encyclopedieberbere.revues.org/1923. Consulté le 28 juin 2017.

Cassar-Pullicino J. 1967 - Ethno-linguistic Aspects of Animals in Malta, Journal of Maltese Studies 4 : $1-68$.

Chaker S. (Ed.) - Encyclopédie Berbère, [En ligne] URL: https://encyclopedieberbere.revues.org/. Consulté le 28 juin 2017.

Colin G.S. 1921 - Le parler arabe du nord de la région de Taza. Bulletin de l'Institut Français D’Archéologie Orientale XVIII : 33-119.

Colin G.S. 1926 - Notes de dialectologie arabe. Étymologies mağribines. Hespéris 6 : 55-82.

Colin G.S. 1927 - Notes de dialectologie arabe. Étymologies magribines II. Hespéris 7 : 85-102.

Colin G.S. 1930 - Étymologies mag̈ribines III. Hespéris 10 : 125-127.

Corominas J. \& Pascual J.A. 1980-1991 - Diccionario crítico etimológico castellano e hispánico. 6 vols. Madrid, Gredos.

Corriente F. 2008 - Dictionary of Arabic and Allied Loanwords: Spanish, Portuguese, Catalan, Galician and Kindred Dialects. Leiden, Boston, Brill.

(DAA) Corriente F. 1997 - A Dictionary of Andalusi Arabic. Leiden, New York, Köln, Brill.

(DAA) Corriente F., Pereira Ch. \& Vicente Á. 2015 - Aperçu grammatical du faisceau dialectal arabe andalou. Perspectives synchroniques, diachroniques et panchroniques. Berlin \& Boston, De Gruyter.

(DAA) Corriente F., Pereira Ch. \& Vicente Á. 2017 - Dictionnaire du faisceau dialectal arabe andalou. Perspectives phraséologiques et étymologiques. Berlin \& Boston, De Gruyter.

(DAF) Prémare A.-L. de et al. 1993-1999 - Dictionnaire arabe-français : établi sur la base de fichiers, ouvrages, enquêtes, manuscrits études et documents divers. 12 vols. Paris, L'Harmattan.

Dallet J.M. 1982 - Dictionnaire kabyle-français. Paris, Société d'études linguistiques et anthropologiques de France.

Direction Générale des Collectivités Locales, Ministère de l'Intérieur, Royaume du Maroc 2015 La région de Tanger - Tétouan - Al Hoceima. Monographie générale : 4.

Dozy R. 1881 - Supplément aux dictionnaires arabes. 2 vols. Leyde, E.J. Brill.

Ferrando I. 1997 - G.S. Colin y los berberismos de árabe andalusí. Revista de estudios de dialectología norteafricana y andalusí 2 : 105-145.

Galand-Pernet P. 1987 - š berbère, phonème, morphème. In Jungraithmayr H. \& Müller W. (eds), Proceedings of the Fourth International Hamito-Semitic Congress: Marburg, 20-22 September, 1983. Amsterdam, Philadelphia, John Benjamins Publishing Company: 381-394.

Goldenberg A. 1999 - La représentation animale au Maroc et ses significations dans l'esprit populaire. In Messaoudi L. \& Zouggari A. (Éds.), Contes et récits. Instruments pédagogiques et produits socioculturels. Kénitra, Université Ibn Tofail, Faculté des Lettres et Sciences Humaine : 49-58.

González Sancho A.J. 2010 - Arquitectura rural en tierra en el norte de Marruecos. Un paisaje cultural en transformación. Tanger, Alcorah Global Industrias Gráficas.

Guerrero, J. (à paraître) - Reflexes of old Arabic*/ğ/ in the Maghrebi Dialects.

Heath J. 2002 - Jewish and Muslim Dialects of Moroccan Arabic. London, Routledge Curzon. 
Heath J. 2006 - Dictionnaire touareg du Mali : tamachek-anglais-français. Paris, Karthala.

Ibáñez E. 1959 - Diccionario español-senhaji (dialecto bereber de Senhaya de Serair). Madrid, Consejo Superior de Investigaciones Científicas.

Kazimisrski A. de B. 1875 - Dictionnaire arabe-français. 4 vols. Caire, Boulac.

Kossmann M. 2013 - The Arabic Influence on Northern Berber. Leiden-Boston, Brill.

Lane E.W. 1863 - An Arabic-English Lexicon. London, Williams and Norgate, [En ligne] URL : http:// www.tyndalearchive.com/tabs/lane/. Consulté le 28 juin 2017.

Laoust E. 1936 - Cours de berbère marocain. Dialectes du Sous du Haut et de l'Anti-Atlas. Paris, Société d'Éditions Géographiques, Maritimes et Coloniales.

Leibring K. 2016 - Animal names. In Hough C. (Ed.), The Oxford Handbook of Names and Naming. Oxford, Oxford University Press : 615-627.

Lévi-Provençal É. 1922 - Textes arabes de l’Ouargha. Dialecte des Jbala (Maroc septentrional). Paris, Édition Ernest Leroux.

Madouni-La Peyre J. 2003 - Dictionnaire arabe algérien-français. Algérie de l'ouest. Paris, l'Asiathèque.

Marçais Ph. 1977 - Esquisse grammaticale de l'arabe maghrébin. Paris, Librairie d'Amérique et d'Orient Adrien Maisonneuve.

Monteil V. 1951 - Contribution à l'étude de la faune du Sahara occidental. Paris, Éditions Larose.

Monteil V. 1952 - Essai sur le chameau au Sahara Occidental. Centre IFAN, Mauritanie.

Mourigh Kh. 2016 - A Grammar of Ghomara Berber (North-West Morocco). Köln, Rüdiger Köppe Verlag.

Moscoso F. 2003 - El dialecto árabe de Chauen (N. De Marruecos). Estudio lingüístico y textos. Cádiz, Universidad de Cádiz.

Naït-Zerrad K. 1998-2002 - Dictionnaire des racines berbères (formes attestées). 3 vols. Paris, Louvain, Peeters.

Raki M. \& Haj Soulami M.B. 2001 - L'élevage caprin, une alternative de développement en zone de montagne ? In : Zouggari A., Vignet-Zunz J. \& Messaoudi L. (Éds.), Les Jbala : Espace et pratique. Kénitra, Université Ibn Tofaïl, Faculté des Lettres et Sciences Humaines : 121-134.

Renisio A. 1932 - Étude sur les dialectes berbères des Beni Iznassen, du Rif et des Senhaja de Sraïr. Paris, Leroux.

Serhoual M. 2001-2002 - Dictionnaire tarifit-français. Thèse de doctorat d'État. Tétouan, Université Abdelmalek Essaâdi.

Simonet F.J. 1888 - Glosario de voces ibéricas y latinas usadas entre los mozárabes. Madrid, Establecimiento tipográfico de Fortanet.

Souag L. 2005 - Notes on the Algerian Arabic dialects of Dellys. Revista de estudios de dialectología norteafricana y andalusí $9: 151-180$.

Vicente Á. 2000 - El dialecto árabe de Anjra (norte de Marruecos). Estudios lingüístico y textos. Zaragoza, Universidad de Zaragoza.

Vicente Á, Caubet D. \& Naciri-Azzouz A. 2017 - La région du Nord-Ouest marocain : Parlers et pratiques sociales et culturelles. Zaragoza, Prensas de la Universidad de Zaragoza. 
Vignet-Zunz J. 1995 - Djebala. Encyclopédie berbère XVI. [En ligne] URL : http:// encyclopedieberbere.revues.org/2176. Consulté le 28 juin 2017.

Vignet-Zunz J. 2014 - Les Jbala du Rif. Des lettrés en montage. Aix-en-Provence, IREMAM.

Westermarck E. 1930 - Wit and Wisdom in Morocco. A Study of Native Proverbs. London, George Routledge \& Sons.

Zarguef A. 2001 - Ighrem, Encyclopédie Berbère XXIV. [En ligne] URL : https:// encyclopedieberbere.revues.org/. Consulté le 28 juin 2017.

\section{NOTES}

1. Pour une discussion sur les ethnonymes et l'histoire de cette région, cf. Vignez-Zunz 1995 et Camps \& Vignez-Zunz 1998.

2. Financés par le projet La montagne et ses savoirs (Rif occidental, Maroc) du PICS IREMAMUniversité de Fès (2013-2015), et le projet Fronteras lingüísticas y factores sociales: perspectivas sincrónicas y diacrónicas de la región del Magreb (MINECO, Espagne), (2012-2015). Et également par une aide à la mobilité prédoctorale en 2015 (MINECO, Espagne).

3. dabbar rāạsu « Il s'est débrouillé seul ».

4. əl-Yard a été documenté par Colin chez les Ghzawa, qui sont aussi des Jbala, avec les sens «tête de bovidé " (DAF 2017 9: 73). Dans l'arabe andalou, le mot Sarad avait le sens de «biens, marchandise » (DAA 2017 : 349).

5. http://www.fao.org/docrep/w3586f/w3586f06.htm et http://om.ciheam.org/om/pdf/ a61/04600112.pdf; [consulté le 21/06/2017].

6. La culture du chanvre est une des activités principales de la région. Comme le signale Bouillot (2010: 95) «l'élevage en pays Jbala est une activité parfaitement adaptée à la gestion de l'économie montagnarde [...] et n'apparaît pas comme une alternative face à des productions plus rentables comme le cannabis ».

7. Cette terminologie a aussi une relation étroite avec les stades du cycle vital de l'animal et sa productivité : reproduction, lait, etc.

8. Dans les variétés jbala, le suffixe latin -š a été spécialement attesté, pour les valeurs de ce suffixe voir Colin 1926, et dans le cas du berbère, Galand-Pernet 1987.

9. Dans la liste ci-dessous on ne va pas inclure les surnoms pour les chiens, les chats et les autres animaux domestiques.

10. Ce schème avec - $\bar{u}$ est utilisé avec des valeurs intensifs et expressifs, c'est pourquoi son usage est plus courant avec des adjectifs (Marçais 1977 : 106-107).

11. La dissimilation de / $\breve{g} />[\mathrm{g}]$ en contact avec des sifflantes qui le suivent a comme résultat des mots connus partout au Maroc comme glas «il s'est assis » et gnāza « enterrement », ou des réalisations plus spécifiques du nord, comme s-semāna lli gāzat « la semaine dernière ». Sur ce phonème et ses réalisations dans les dialectes maghrébins, voir Guerrero (à paraître).

12. Selon González Sancho (2010: 70): «La technique de construction en terre est une connaissance appréhendée d'une génération à l'autre. C'est un patrimoine culturel intangible. À la génération suivante, la transmission de cette maîtrise, de ce savoir-faire... sera perdue de manière irréversible ».

13. Certains termes sont employés aussi pour les humains. 


\section{RÉSUMÉS}

L'élevage est l'une des activités de l'économie traditionnelle et de subsistance dans la région des Jbala. Cependant, les changements socio-économiques qui ont lieu dans le nord-ouest marocain ont comme conséquence la disparition d'un patrimoine culturel dont la langue quotidienne pour nommer les techniques ou l'environnement fait partie. Ce travail présente un petit corpus du lexique portant sur l'élevage, recueilli sur place avec les paysan/nes impliqué/es dans cette activité. Nous nous sommes focalisées sur certains termes très intéressants au moyen d'une analyse linguistique ainsi que de données ethnographiques.

Livestock is one of the activities of the traditional subsistence economy in the Jbala region. Nevertheless, socio-economic changes taking place in Northwestern Morocco are displacing this activity and along with it, the rich vocabulary related to it. This research discusses a small corpus of lexicon related to livestock gathered in situ with the peasants involved in this activity. We have focused on several interesting terms by providing a linguistic analysis and some ethnographical data.

\section{INDEX}

Mots-clés : élevage, Jbala, Ghomara, arabe marocain, ethnolinguistique

Keywords : livestock, Jbala, Ghomara, Moroccan Arabic, ethnolinguistics

\section{AUTEURS}

\section{AMINA NACIRI-AZZOUZ}

Doctorante, Universidad de Zaragoza, Calle Pedro Cerbuna, 12, Facultad de Filosofía y Letras Universidad de Zaragoza, 50009 Zaragoza - Espagne

anaciri@unizar.es

\section{ÁNGELES VICENTE}

Maître de conférences (HDR), Universidad de Zaragoza, Calle Pedro Cerbuna, 12, Facultad de Filosofía y Letras - Universidad de Zaragoza, 50009 Zaragoza - Espagne

mavicen@unizar.es 